\title{
Radiation of a bunch in a waveguide with a semibounded anisotropic dielectric
}

\author{
Tatiana Yu. Alekhina, ${ }^{*}$ Andrey V. Tyukhtin, ${ }^{\dagger}$ and Sergey N. Galyamin \\ Saint Petersburg State University, 7/9 Universitetskaya nab., St. Petersburg, 199034, Russia
}

(Received 30 November 2018; published 10 April 2019)

\begin{abstract}
We analyze the electromagnetic field (EMF) of a small bunch that moves uniformly in a circular metal waveguide and crosses the boundary between two areas: a vacuum area and an area loaded with an anisotropic homogeneous nondispersive dielectric characterized by a diagonal permittivity tensor. Two cases are studied in detail: the bunch flies from a vacuum into the dielectric and, inversely, from the dielectric into a vacuum. In the second case, so-called Cherenkov-transition radiation (CTR) can be generated in the vacuum area of the waveguide. Exact expressions for the EMF components are obtained and investigated. A comparison between the present problems and the analogous problems in the case of an isotropic dielectric is performed. We discuss some benefits occurring from using the anisotropic dielectric. For example, anisotropic dielectric allows more efficient generation of radiation compared to the isotropic dielectric for the same bunch and geometry parameters.
\end{abstract}

DOI: 10.1103/PhysRevAccelBeams.22.041301

\section{INTRODUCTION}

Investigation of radiation produced by charged particle bunches in dielectrically loaded waveguides is important for dielectric wakefield acceleration (DWA) technique and other areas of accelerators and beam physics. As for the DWA scheme, many theoretical and experimental papers [1-5] demonstrated high efficiency of dielectric accelerating structures and stability of wakefield parameters. These papers described techniques for structures manufacturing as well.

Recently, there was a great interest in having a source of short intense pulses of Terahertz $(\mathrm{THz})$ radiation. One of beam-based methods is using a metallic beam pipe with small corrugation [6,7]. DWA structures are also considered as promising candidates for generation of radiation in the Terahertz range [5,8-12]. It is notable that direct experimental observation of wakefield effects in a $\mathrm{THz}$ regime was performed in a planar diamond accelerating structure [13] and in a cylindrical dielectric-lined waveguides with the use of tubes fabricated from fused silica [14] and chemical-vapor deposition diamond [15].

Anisotropy of materials used in such DWA structures is one possible way to increase the generated wakefield $[16,17]$. The case of an infinitely long rectangular structure with an

\footnotetext{
*tanya_alekhina@mail.ru, t.alehina@spbu.ru

tyukhtin@bk.ru
}

Published by the American Physical Society under the terms of the Creative Commons Attribution 4.0 International license. Further distribution of this work must maintain attribution to the author(s) and the published article's title, journal citation, and DOI. anisotropic natural material was analyzed in [17] but benefits of anisotropy were not properly discussed. Apart from natural anisotropic materials (crystals and various thermal insulators), the anisotropy can be artificially realized, for example, with layered isotropic materials (disks or rings) [18] or various carbon-based composite materials [19].

It should be noticed that beam field structure in an infinite regular waveguide loaded with an anisotropic dielectric was considered in a series of papers and books [1,16,17,20,21]. Cherenkov radiation (CR) in the form of wakefield can be generated in such structures similarly to the well-known case of the waveguide with the isotropic dielectric loading. Underline that the authors pointed out the possibility of significant increasing the acceleration gradient because of anisotropy of the waveguide filling [1,16,20,21].

One of the interesting problems consists in analysis of the influence of the transversal boundary on the wakefield when the bunch flies into (or flies from) the dielectric structure. Such problems were considered previously only for isotropic media. The semi-infinite waveguide having isotropic filling and the metal end was analyzed in papers $[22,23]$. Problems with an interface between different homogenous isotropic dielectrics in the waveguide were studied in our previous papers [24-26]. We also analyzed such problems for the case when one semi-infinite part of the waveguide has an isotropic dielectric layer and a vacuum channel $[27,28]$. In particular, it was shown that the area of the boundary influence can be decreased if the dielectric with relatively large permittivity is used. However, the analogous problem with an anisotropic dielectric has not been considered although it is a promising material for generating high field gradients. 
Here we investigate the influence of anisotropy on radiation of a bunch in the dielectrically loaded waveguide with a transversal border. We consider two variants: the case of the bunch flying into the dielectric and the opposite case when the bunch flies from dielectric into a vacuum.

\section{THE PROBLEM STATEMENT}

We consider a small bunch $q$ moving uniformly with a velocity $\vec{V}=c \beta \vec{e}_{z}$ ( $c$ is the light velocity) in a metal circular waveguide of the radius $a$ and intersecting the boundary (at $z=0$ ) between two areas: a vacuum area and an area loaded with an anisotropic homogeneous nondispersive dielectric (Fig. 1). The bunch moves along the optical axis of the dielectric, which coincides with the $z$-axis of the cylindrical frame of reference $r, \phi, z$. The dielectric is described by a diagonal permittivity tensor $\hat{\varepsilon}$ with components:

$$
\varepsilon_{11}=\varepsilon_{22}=\varepsilon_{r}, \quad \varepsilon_{33}=\varepsilon_{z}, \quad \varepsilon_{i j}=0 \text { for } i \neq j .
$$

We consider only the situation when $\varepsilon_{z}>0$ and $\varepsilon_{r}>0$ because this usually takes place in practice in frequency ranges without dispersion (negative values of $\varepsilon_{z}$ and $\varepsilon_{r}$ can be observed only in the frequency ranges with essential dispersion). The bunch is characterized by a distribution along the $z$-axis and a negligible thickness, i.e., the charge density is

$$
\rho=q \delta(r) \eta(\zeta) /(2 \pi r), \quad \int_{-\infty}^{\infty} \eta(\zeta) d \zeta=1, \quad \zeta=z-c t \beta .
$$

The most common example is the bunch having the Gaussian distribution $\eta(\zeta)=\exp \left(-\zeta^{2} /\left(2 \sigma^{2}\right)\right) /(\sqrt{2 \pi} \sigma)$, where $\sigma$ is the r.m.s. half-length of the bunch. The center of the bunch intersects the boundary at the moment $t=0$.

We investigate two cases: the bunch flies from a vacuum into the dielectric, and, inversely, from the dielectric into a vacuum. The expressions for the field components can be written in the form

$$
E_{r, z 1,2}=E_{r, z 1,2}^{q}+E_{r, z 1,2}^{b}, \quad H_{\phi 1,2}=H_{\phi 1,2}^{q}+H_{\phi 1,2}^{b},
$$

where subscripts 1 and 2 relate to the areas $z<0$ and $z>0$, respectively. The first summands in (2) are for the "forced" field [29], which is the field of the bunch in the regular waveguide. It can contain $\mathrm{CR}$ under certain conditions.

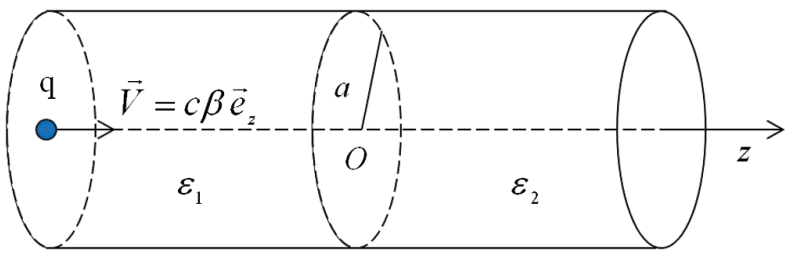

FIG. 1. Geometry of the problem.
The second summands in (2) are the "free" field components [29]. The "free" field is connected with the influence of the boundary. It includes transition radiation (TR) and, under certain conditions, the Cherenkov-transition radiation (CTR) [24,25]. The analytical solutions for the forced and free fields are given in the form of a decomposition in an infinite series of normal modes.

We analyze the exact solution for the field components of each mode in two different cases using the methods of the complex variable function theory. Note that such methods were applied in our previous papers for different problems with sectionally homogeneous semi-infinite media and waveguides [30-32] including the analogous problems with an isotropic dielectric [24,25]. Further, we will make also a comparison between these two problems and will show some benefits from using the anisotropic dielectric as opposed to the isotropic one.

\section{CR IN THE ANISOTROPIC DIELECTRIC}

First, we study the modes of the "forced" field in (2) that is the field of a bunch in a regular waveguide. The forced field components in the anisotropic dielectric are written in the form:

$$
\begin{aligned}
\left\{\begin{array}{c}
E_{r}^{q} \\
H_{\phi}^{q}
\end{array}\right\}= & \frac{2 q \beta^{2} c}{\pi a^{3}} \sum_{n=1}^{\infty} \frac{\chi_{n} J_{1}\left(\chi_{n} r / a\right)}{J_{1}^{2}\left(\chi_{n}\right)} \\
& \times \int_{-\infty}^{+\infty} d \omega \frac{\eta_{\omega}(\omega) \exp [i \zeta / c \beta]}{\beta^{2} c^{2} k_{z}^{2}-\omega^{2}}\left\{\begin{array}{c}
e_{r}^{q} \\
h_{\phi}^{q}
\end{array}\right\}, \\
E_{z}^{q}= & \frac{2 i q \beta^{2}}{\pi a^{4}} \sum_{n=1}^{\infty} \frac{J_{0}\left(\chi_{n} r / a\right)}{J_{1}^{2}\left(\chi_{n}\right)} \\
& \times \int_{-\infty}^{+\infty} d \omega \frac{\omega \eta_{\omega}(\omega) \exp [i \zeta / c \beta]}{\beta^{2} c^{2} k_{z}^{2}-\omega^{2}} e_{z}^{q},
\end{aligned}
$$

where $e_{r}^{q}=\left(\beta \varepsilon_{z}\right)^{-1}, e_{z}^{q}=\varepsilon_{z}^{-1}\left(\beta^{-2}-\varepsilon_{r}\right), h_{\phi}^{q}=\varepsilon_{r}\left(\varepsilon_{z}\right)^{-1}$, $k_{\mathrm{z}}=\left(\varepsilon_{r} \omega^{2}-\varepsilon_{r} \varepsilon_{z}^{-1} \omega_{n}^{2}\right)^{1 / 2} c^{-1}, \omega_{n}=\chi_{n} c / a, \chi_{n}$ is the $n$th zero of the Bessel function $\left(J_{0}\left(\chi_{n}\right)=0\right)$, and $\eta_{\omega}(\omega)$ is the Fourier transform of the longitudinal distribution $\eta(\zeta)$ normalized to the Fourier transform of a point charge density (for example, for the bunch with the Gaussian distribution $\left.\eta_{\omega}(\omega)=\exp \left[-\omega^{2} \sigma^{2}\left(2 \beta^{2} c^{2}\right)^{-1}\right]\right)$. Note that the forced field in a vacuum is obtained from Eq. (3) with the substitution $\varepsilon_{z}=\varepsilon_{r}=1$.

The investigation of Eq. (3) is based on the complex variable function theory. The integrand singularities in (3) are the poles on the complex plane of $\omega$. If these poles are situated on the real axis then CR is generated in the dielectric. Therefore, the condition for $\mathrm{CR}$ generation is

$$
\frac{\varepsilon_{z}}{\varepsilon_{r}}\left(\varepsilon_{r} \beta^{2}-1\right) \geq 0 .
$$


Since we consider only the situation when $\varepsilon_{z}>0$ and $\varepsilon_{r}>0$ then $\mathrm{CR}$ is generated under the condition

$$
\beta \geq \beta_{C}=\varepsilon_{r}^{-1 / 2} .
$$

The CR frequencies are

$$
\omega_{0 n}=\omega_{n} \beta \varepsilon_{z}^{-1 / 2}\left(\beta^{2}-\varepsilon_{r}^{-1}\right)^{-1 / 2} .
$$

Note that analogous investigation for the isotropic dielectric with the permittivity $\varepsilon=\varepsilon_{r}>0$ [25] gives the real frequencies of $\mathrm{CR} \tilde{\omega}_{0 n}=\beta \omega_{n}\left(\varepsilon_{r} \beta^{2}-1\right)^{-1 / 2}$ under the condition $\beta \geq \varepsilon_{r}^{-1 / 2}$. We see that

$$
\omega_{0 n} / \tilde{\omega}_{0 n}=\sqrt{\varepsilon_{r} / \varepsilon_{z}}
$$

So, frequencies of the radiated waves are larger than the ones in the isotropic dielectric if $\varepsilon_{r}>\varepsilon_{z}$.

Note that the poles $\pm \omega_{0 n}$ (6) are slightly shifted downward from the real axis if small losses are taken into account. Therefore, the integration path goes along the real axis above these poles. Their contributions (i.e., sum of residues) determine $\mathrm{CR}$ :

$$
\begin{aligned}
\left\{\begin{array}{c}
E_{r}^{\mathrm{CR}} \\
H_{\phi}^{\mathrm{CR}}
\end{array}\right\}= & \frac{4 q}{a^{2}}\left\{\begin{array}{c}
e_{r}^{\mathrm{CR}} \\
h_{\phi}^{\mathrm{CR}}
\end{array}\right\} \sum_{n=1}^{\infty} \frac{\chi_{n} J_{1}\left(\chi_{n} r / a\right)}{J_{1}^{2}\left(\chi_{n}\right)} \eta_{\omega}\left(\omega_{0 n}\right) \\
& \times \sin \left[\omega_{0 n}\left(t-z(c \beta)^{-1}\right)\right] \Theta\left(t-z(c \beta)^{-1}\right), \\
E_{z}^{\mathrm{CR}}= & \frac{4 q}{a^{2} \varepsilon_{z}} \sum_{n=1}^{\infty} \frac{J_{0}\left(\chi_{n} r / a\right)}{J_{1}^{2}\left(\chi_{n}\right)} \eta_{\omega}\left(\omega_{0 n}\right) \\
& \times \cos \left[\omega_{0 n}\left(t-z(c \beta)^{-1}\right)\right] \Theta\left(t-z(c \beta)^{-1}\right),
\end{aligned}
$$

where $e_{r}^{\mathrm{CR}}=\left[\varepsilon_{r} \varepsilon_{z}\left(\varepsilon_{r} \beta^{2}-1\right)\right]^{-1 / 2}, h_{\phi}^{\mathrm{CR}}=\beta\left[\varepsilon_{z}\left(\beta^{2}-\varepsilon_{r}^{-1}\right)\right]^{-1 / 2}$, and $\theta(x)$ is the Heaviside step function.

In comparison with the case of isotropic dielectric, we have

$\frac{E_{r}^{\mathrm{CR}}}{\tilde{E}_{r}^{\mathrm{CR}}}=\frac{1}{\sqrt{\left(\varepsilon_{r} \varepsilon_{z}\right)}}, \quad \frac{E_{z}^{\mathrm{CR}}}{\tilde{E}_{z}^{\mathrm{CR}}}=\frac{\varepsilon_{r}}{\varepsilon_{z}}, \quad \frac{H_{\phi}^{\mathrm{CR}}}{\tilde{H}_{\phi}^{\mathrm{CR}}}=\sqrt{\frac{\varepsilon_{r}}{\varepsilon_{z}}}$,

where magnitudes marked with tildes are related to the isotropic case. As one can see, using anisotropic dielectric allows increasing the radiation intensity without any change of the bunch velocity or the waveguide radius. The measurement of the CR frequency shift (7) and the CR amplitude (9) would be also used for the analysis of the anisotropic dielectric properties and for estimation of the longitudinal $\varepsilon_{z}$ and transversal $\varepsilon_{r}$ components of the permittivity tensor.

\section{THE CASE OF THE BUNCH FLYING FROM A VACUUM INTO THE ANISOTROPIC DIELECTRIC}

The exact expressions for the free field components in the case when the bunch flies from a vacuum $\left(\varepsilon_{1}=1\right)$ into the anisotropic dielectric with $\varepsilon_{2}=\hat{\varepsilon}(1)$ are written in the form

$$
\begin{aligned}
\left\{\begin{array}{c}
E_{r 1,2}^{b} \\
H_{\phi 1,2}^{b}
\end{array}\right\}= & -\frac{2 q \beta}{\pi a^{3}} \sum_{n=1}^{\infty} \frac{\chi_{n} J_{1}\left(\chi_{n} r / a\right)}{J_{1}^{2}\left(\chi_{n}\right)} \int_{-\infty}^{+\infty}\left\{\begin{array}{c}
e_{r 1,2}^{b} \\
h_{\phi 1,2}^{b}
\end{array}\right\} \\
& \times B_{n 1,2} \eta_{\omega}(\omega) \exp \left[i\left(k_{z 1,2}|z|-\omega t\right)\right] d \omega, \\
E_{z 1,2}^{b}= & -\frac{2 i q}{\pi a^{4}} \sum_{n=1}^{\infty} \frac{\chi_{n}^{2} J_{0}\left(\chi_{n} r / a\right)}{J_{1}^{2}\left(\chi_{n}\right)} \\
& \times \int_{-\infty}^{+\infty} \frac{e_{z 1,2}^{b}}{\omega} B_{n 1,2} \eta_{\omega}(\omega) \\
& \times \exp \left[i\left(k_{z 1,2}|z|-\omega t\right)\right] d \omega,
\end{aligned}
$$

where $h_{\phi 1,2}^{b}=e_{z 1}^{q}=1, e_{z 2}^{b}=\varepsilon_{z}^{-1}, e_{r 1}^{q}=-c k_{z 1} \omega^{-1}, e_{r 2}^{b}=$ $c k_{z 2}\left(\omega \varepsilon_{r}\right)^{-1}$,

$B_{n 1}=\frac{\varepsilon_{r}}{\varepsilon_{z}\left(k_{z 1}+\varepsilon_{r} k_{z 2}\right)}\left[\frac{1}{\left(c \beta k_{z 2}+\omega\right)}+\frac{\varepsilon_{z}\left(\varepsilon_{r} \omega-c \beta k_{z 2}\right)}{\varepsilon_{r}\left(c^{2} \beta^{2} k_{z 1}^{2}-\omega^{2}\right)}\right]$,

$B_{n 2}=\frac{\varepsilon_{r}}{\varepsilon_{z}\left(k_{z 1}+\varepsilon_{r} k_{z 2}\right)}\left[\frac{\varepsilon_{z}}{\left(c \beta k_{z 1}-\omega\right)}-\frac{\left(\omega+\varepsilon_{r} c \beta k_{z 1}\right)}{\left(c^{2} \beta^{2} k_{z 2}^{2}-\omega^{2}\right)}\right]$,

$k_{\mathrm{z} 1}=\frac{\sqrt{\omega^{2}-\omega_{n}^{2}}}{c}, \quad k_{\mathrm{z} 2}=\frac{\sqrt{\varepsilon_{r}\left(\varepsilon_{z} \omega^{2}-\omega_{n}^{2}\right)}}{\sqrt{\varepsilon_{z}} c}$,

and $\operatorname{Im} k_{\mathrm{z} 1,2}>0$ (this condition means that the waves outgoing from the boundary decrease with increase in the distance $|z|$ if we take into account small dissipation in the medium). Asymptotic expressions for the free field components of each mode Eq. (10), (11) are obtained with the steepest descent technique. Note that the procedure is similar to one developed for different situations in our previous papers $[24,30]$. So, here we only pay attention to the main points.

The first step in such research is to study the singularities of integrands in (10) in a complex plane of $\omega$. One can show that integrands have the following singularities: four branch points from radicals (11) $\pm \tilde{\omega}_{n}^{(1)}= \pm \omega_{n}-\mathrm{i} \delta_{1}$ and $\pm \tilde{\omega}_{n}^{(2)}=$ $\pm \omega_{n} / \sqrt{\varepsilon_{z}}-\mathrm{i} \delta_{2}$; two poles on the imaginary axis $(\omega)$ : $\pm \omega_{0 n}^{(1)}= \pm \mathrm{i} \beta \omega_{n}\left(1-\beta^{2}\right)^{-1 / 2}$; two poles $\pm \omega_{0 n}= \pm \omega_{0 n}-$ $i \delta_{3}$ [see Eq. (6)] if the condition (5) is fulfilled and on the imaginary axis otherwise. Here $\delta_{1}, \delta_{2}, \delta_{3}$ are positive infinitesimal quantities. Therefore, the singularities are situated below the real axis. Figure 2 shows all the singularities on the complex plane of $\omega$ and the comparative disposition of the integration path that goes along the upper bank of the branch cuts defined by the equations $\operatorname{Re} \sqrt{\omega^{2}-\omega_{n}^{2}}=0, \operatorname{Re} \sqrt{\omega^{2} \varepsilon_{z}-\omega_{n}^{2}}=0$. 


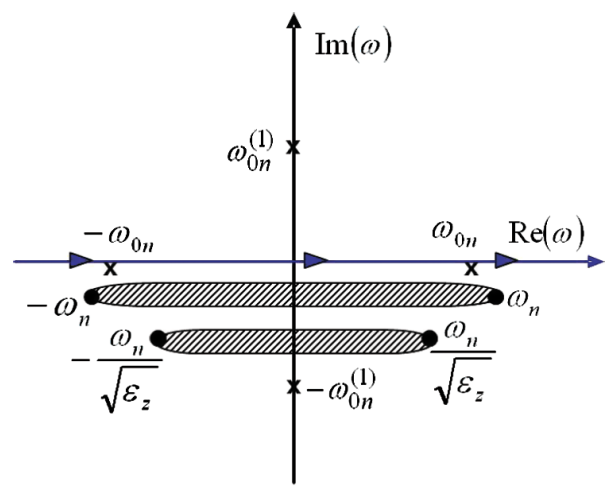

FIG. 2. Disposition of the singularities of the integrands, branch cuts and integration path in a complex plane of $\omega$ for the free field components for $\beta>\varepsilon_{r}^{-1 / 2}$. The poles and the branch points are shown with crosses and circles, correspondingly.

The most important results of the investigation of the free field components concern the contributions of the poles $\pm \omega_{0 n}$ which are determined by the same Eq. (6) as for the forced field. In the vacuum area, there are no such contributions, i.e., CR does not penetrate through the boundary and the free field components correspond to TR as well as in the case of isotropic dielectric [24,25]. In the dielectric, these contributions are determined by formulas (8) but taken with an opposite sign. So, there is a compensation for the forced field with a part of the free field in the domain $z<z_{1}$ near the boundary,

$$
z_{1}=c t /\left(\beta \varepsilon_{r}\right) .
$$

Note that the point $z_{1}$ is determined by the group velocity $v_{g 1}=c / \beta \varepsilon_{r}$ in a regular waveguide as well as in the case of isotropic dielectric $[24,25]$. As a result, there is the area where the wave field practically coincides with the wakefield in the regular waveguide $\left(z_{1}<z<c \beta t\right)$. There is also the area where the boundary influence is principal $\left(z<z_{1}\right)$. Note that the first area is large if the transversal component of the permittivity tensor $\varepsilon_{r}$ is a large value.

Figure 3 illustrates the effect under consideration. Note that the total field (continuous line 1) is obtained numerically from Eq. (10) and (11) with the method based on a transformation of the initial integration path in a complex plane of $\omega$ used before in our previous works [24,30]. For the used parameters, we have $z_{1}=7.5 a$. As one can see, field in vacuum area $(z<0)$ is only TR which is several orders of magnitude smaller compared to the field in the dielectric area $(z>0)$. For $z<z_{1}$, the effect of compensation of the forced field takes place and the boundary strongly affects the wakefield. For $z_{1}<z<c \beta$, two curves shown in Fig. 3 coincide. In this area, EMF in the waveguide with transversal boundary coincides with EMF in a waveguide with uniform dielectric filling. Recall that, in the anisotropic dielectric, the magnitude of $\mathrm{CR}$ is proportional to $\varepsilon_{r} \varepsilon_{z}^{-1}$.

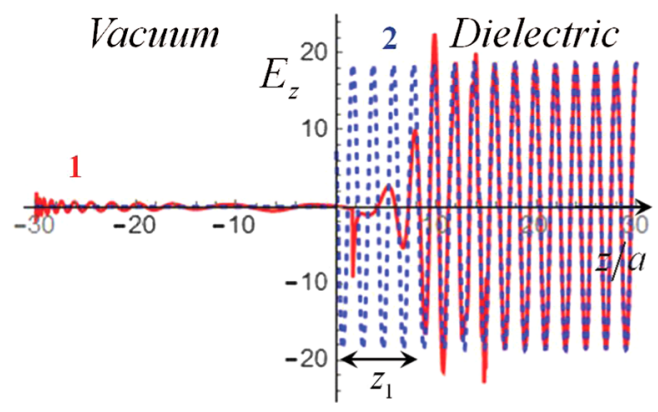

FIG. 3. Dependence of longitudinal component $E_{z}$ (in units of $q / a^{2}$ ) of the first mode of the total field (continuous line 1) and the forced field (dashed line 2) on distance $z / a$ for $c t / a=30$ and the velocity $\gamma=\left(1-\beta^{2}\right)^{-1 / 2}=100 ; \varepsilon_{r}=4, \varepsilon_{z}=0.8$, $\sigma=0$ and $x_{\mathrm{r}}=a \omega_{\mathrm{r}} / c=4$, where $\omega_{\mathrm{r}}$ is a reference frequency. Observation point is on the waveguide axis, $r=0$.

This means that, if $\varepsilon_{z}<\varepsilon_{r}$, then the radiation is more intensive in comparison with the case of the isotropic dielectric with $\varepsilon_{z}=\varepsilon_{r}$. The "compensation domain" where the radiation field is small is the same as in the isotropic dielectric.

\section{THE CASE OF THE BUNCH FLYING FROM THE ANISOTROPIC DIELECTRIC INTO A VACUUM}

When the bunch flies from the anisotropic dielectric with $\varepsilon_{1}=\hat{\varepsilon}(1)$ into a vacuum $\left(\varepsilon_{2}=1\right)$, the exact expressions for the free field components are written in the same form (10), where $h_{\phi 1,2}^{b}=e_{z 2}^{q}=1, \quad e_{z 1}^{b}=\varepsilon_{z}^{-1}, \quad e_{r 1}^{b}=-c k_{z 1}\left(\omega \varepsilon_{r}\right)^{-1}$, $e_{r 2}^{q}=c k_{z 2} \omega^{-1}$,

$B_{n 1}=\frac{-\varepsilon_{r}}{k_{z 1}+\varepsilon_{r} k_{z 2}}\left[\left(c \beta k_{z 2}+\omega\right)^{-1}+\frac{\left(\omega-c \beta \varepsilon_{r} k_{z 2}\right)}{\varepsilon_{z}\left(c^{2} \beta^{2} k_{z 1}^{2}-\omega^{2}\right)}\right]$,

$B_{n 2}=\frac{-\varepsilon_{r}}{\varepsilon_{z}\left(k_{z 1}+\varepsilon_{r} k_{z 2}\right)}\left[\frac{1}{\left(c \beta k_{z 1}-\omega\right)}-\frac{\left(\omega \varepsilon_{z}-c \beta k_{z 1}\right)}{\left(c^{2} \beta^{2} k_{z 2}^{2}-\omega^{2}\right)}\right]$,

$k_{\mathrm{z} 1}=\frac{\sqrt{\varepsilon_{r}\left(\varepsilon_{z} \omega^{2}-\omega_{n}^{2}\right)}}{\sqrt{\varepsilon_{z}} c}, \quad k_{\mathrm{z} 2}=\frac{\sqrt{\omega^{2}-\omega_{n}^{2}}}{c}$.

The investigations for the free field components (10), (13) are made similarly to the previous case. If $\beta \geq \beta_{C}$ (5), then $\mathrm{CR}$ emerges in the dielectric. Analyses of the free field integrands (13) show that the contributions of poles $\pm \omega_{0 n}$ (6) can give the CTR, i.e. the reflected and transmitted waves of CR both in the dielectric and in the vacuum area of the waveguide under certain condition. The most interesting effect concerns the situation when CR penetrates through the boundary into the vacuum area.

In a vacuum, expressions for the CTR components of the free field are the following: 


$$
\begin{aligned}
\left\{\begin{array}{c}
E_{r 2}^{\mathrm{CTR}} \\
H_{\phi 2}^{\mathrm{CTR}}
\end{array}\right\}= & \frac{4 q}{a^{2}}\left\{\begin{array}{l}
e_{r 2}^{\mathrm{CTR}} \\
h_{\phi 2}^{\mathrm{CRR}}
\end{array}\right\} \sum_{n=1}^{\infty} \frac{J_{1}\left(\chi_{n} r / a\right)}{J_{1}^{2}\left(\chi_{n}\right)} \eta_{\omega}\left(\omega_{0 n}\right) \\
& \times \sin \left[\omega_{0 n}\left(z \Psi c \beta^{-1}-t\right)\right] \Theta\left(z_{2}-z\right), \\
E_{z 2}^{\mathrm{CTR}}= & \frac{4 q}{a^{2}} e_{z 2}^{\mathrm{CTR}} \sum_{n=1}^{\infty} \frac{J_{0}\left(\chi_{n} r / a\right)}{J_{1}^{2}\left(\chi_{n}\right)} \eta_{\omega}\left(\omega_{0 n}\right) \\
& \times \cos \left[\omega_{0 n}\left(t-z \Psi c \beta^{-1}\right)\right] \Theta\left(z_{2}-z\right),
\end{aligned}
$$

where

$$
\begin{aligned}
\Psi & =\left[\beta^{2}\left(1-\varepsilon_{z}\right)+\varepsilon_{z} \varepsilon_{r}^{-1}\right]^{1 / 2}, \quad e_{z 2}^{\mathrm{CTR}}=2\left(\Psi \varepsilon_{r}+1\right)^{-1}, \\
e_{r 2}^{\mathrm{CTR}} & =2 \Psi\left(\Psi \varepsilon_{r}+1\right)^{-1}\left[\varepsilon_{z}\left(\beta^{2}-\varepsilon_{r}^{-1}\right)\right]^{-1 / 2}, \\
h_{\phi 2}^{\mathrm{CTR}} & =2 \beta\left(\Psi \varepsilon_{r}+1\right)^{-1}\left[\varepsilon_{z}\left(\beta^{2}-\varepsilon_{r}^{-1}\right)\right]^{-1 / 2} .
\end{aligned}
$$

This field exists only under conditions

$$
\beta_{C}<\beta<\beta_{\mathrm{CT}} .
$$

Here, the lower threshold $\beta_{C}$ is connected with condition of the CR generation (5). The upper threshold

$\beta_{\mathrm{CT}}= \begin{cases}\varepsilon_{r}^{-1 / 2}\left(1-\varepsilon_{z}^{-1}\right)^{-1 / 2}, & \varepsilon_{z} \geq 1+\left(\varepsilon_{r}-1\right)^{-1} \\ 1, & \varepsilon_{z}<1+\left(\varepsilon_{r}-1\right)^{-1}\end{cases}$

is explained by total internal reflection of $\mathrm{CR}$ waves from the boundary. This takes place at $\beta>\beta_{\mathrm{CT}}$ if $\beta_{\mathrm{CT}}<1$, otherwise, if $\beta_{\mathrm{CT}}=1$ (16), all waves of $\mathrm{CR}$ penetrate through the boundary and CTR can be generated for ultrarelativistic bunches at some parameters of the problem.

In a vacuum, the CTR exists in the area $z<z_{2}=c t \Psi / \beta$. The length of this zone $z_{2}$ increases with group velocity of waveguide waves $V_{g 2}=c \Psi / \beta$. Note that similar effect takes place in the isotropic dielectric with $\varepsilon=\varepsilon_{r}$. The difference is that the upper threshold is less than unity $\tilde{\beta}_{\mathrm{CT}}=\left(\varepsilon_{r}-1\right)^{-1 / 2}<1$ for $\varepsilon_{r}>2$ as opposed to the case with the anisotropic dielectric when the frequency range of the CTR generating can be increased right up to the ultrarelativistic situation.

Figure 4 illustrates the effect under consideration for the parameters of the problem when there is no CTR in the isotropic case with $\varepsilon=\varepsilon_{r}$. In a vacuum, the total wave field consists of TR and the CTR (transmitted wave of CR). As one can see, the CTR can be dominant at $\beta_{C}<\beta<\beta_{\mathrm{CT}}$ [here $\beta_{\mathrm{CT}}=1$ (16)] in some domain near the boundary $z<z_{2}$.

Next, we consider the time averaged energy flux of the CTR passing through the cross section of the waveguide:

$$
\bar{\Sigma}^{\mathrm{CTR}}=\frac{c}{4 \pi} \frac{1}{T} \int_{0}^{T} d t \int_{0}^{2 \pi} d \phi \int_{0}^{a} r d r H_{\phi}^{\mathrm{CTR}} E_{r}^{\mathrm{CTR}}
$$

where $H_{\phi}^{\mathrm{CTR}}, E_{r}^{\mathrm{CTR}}$ are defined by Eq. (14). Here, time averaging is made by a interval that is much larger than all

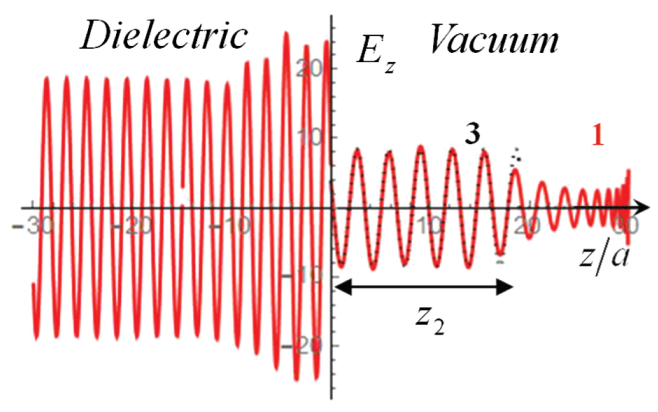

FIG. 4. Dependence of longitudinal component $E_{z}$ (in units of $q / a^{2}$ ) of the first mode of the total field (continuous line 1) and the CTR (dotted line 3 ) on distance $z / a$ for the same parameters of the problem as in Fig. 3.

periods of oscillations of the CTR modes. Thus, the values $\bar{\Sigma}^{\text {CTR }}$ (17) include a double sum of the products of $n$ and $m$ modes. However, the orthogonality of Bessel's functions leads to vanishing of all of the terms with $n \neq m$. As a result, we obtain the following exact expression:

$$
\bar{\Sigma}^{\mathrm{CTR}}=\frac{q^{2} c}{a^{2}} \bar{\Sigma} \sum_{n=1}^{\infty} \frac{\eta_{\omega}\left(\omega_{0 n}\right)}{J_{1}^{2}\left(\chi_{n}\right)},
$$

where

$$
\bar{\Sigma}=8 \beta \varepsilon_{r} \Psi \varepsilon_{z}\left(\Psi \varepsilon_{r}+1\right)^{2}\left(\beta^{2} \varepsilon_{r}-1\right)^{-1} .
$$

Note that the sum in (18) consists of the finite number of terms for the real bunches having finite length $2 \sigma$. For example, for the bunch with the Gaussian distribution we have $\eta_{\omega}\left(\omega_{0 n}\right)=\exp \left[-\omega_{0 n}^{2} \sigma^{2}\left(2 \beta^{2} c^{2}\right)^{-1}\right]$.

In Fig. 5, behavior of the value $\bar{\Sigma}(19)$ is shown at various parameters of the problem. As one can see, $\bar{\Sigma}$ tends to infinity at the lower threshold $\beta \rightarrow \beta_{C}$ as well as the energy flux of the CR [see Eq. (8)]. This effect also takes place for the isotropic dielectric with $\varepsilon=\varepsilon_{r}$.

For the isotropic dielectric in the case $\varepsilon_{r}>2$, the upper threshold is always less than unity $\tilde{\beta}_{\mathrm{CT}}=\left(\varepsilon_{r}-1\right)^{-1 / 2}<1$. This situation is illustrated in Fig. 5 (top, line 4): line 4 is truncated at $\beta=\tilde{\beta}_{\mathrm{CT}} \approx 0.58$. The cases with the anisotropic dielectric Fig. 5 (top, lines 1, 2, 3 and bottom, lines 1,2) and for the isotropic one at $\varepsilon_{r}<2$ (Fig. 5, bottom, line 4) show that the CTR can exist for ultrarelativistic bunches with $\beta \rightarrow 1$. However, the situation with $\beta_{\mathrm{CT}}<1$ for the anisotropic case is also possible (see line 3 in Fig. 5, bottom). It should be also noted that the energy flux of the CTR in the case of the anisotropic dielectric $\bar{\Sigma}$ differs by the magnitude from the energy flux of the CTR in the isotropic case $\bar{\Sigma}$ :

$$
\bar{\Sigma} \approx \tilde{\bar{\Sigma}}_{\epsilon_{r}} \epsilon_{z}^{-1} .
$$

Therefore, the radiation can be more significant for $\varepsilon_{z}<\varepsilon_{r}$ at the same other parameters of the problem which is illustrated in Fig. 5 (top and bottom, lines 1,2). 

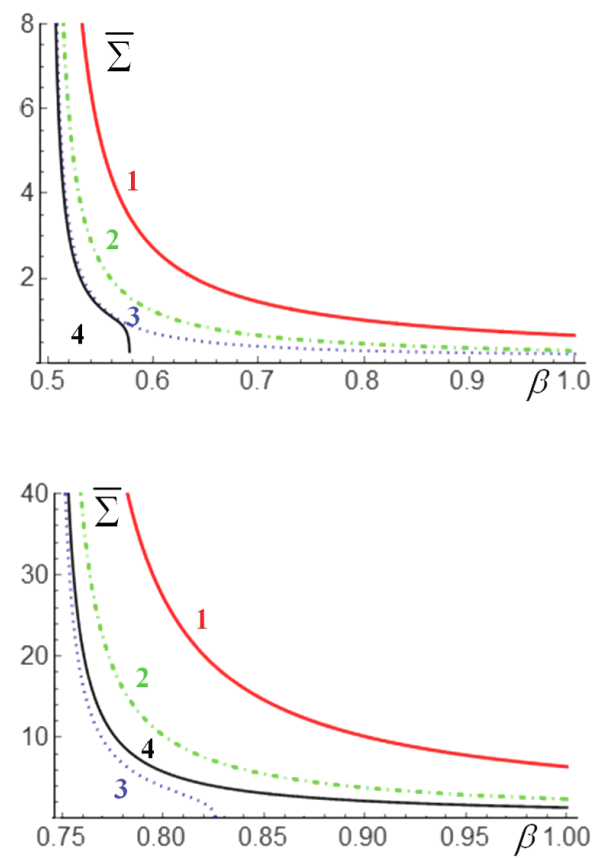

FIG. 5. Dependence of the energy flux of the CTR $\bar{\Sigma}$ on the velocity $\beta$ at different parameters: $\varepsilon_{r}=4$ (top) and $\varepsilon_{r}=1.8$ (bottom); $\varepsilon_{z}=0.2$ (thick continuous red line 1), $\varepsilon_{z}=0.8$ (dashed green line 2), $\varepsilon_{z}=2.2$ (dotted blue line 3 ), and the isotropic case $\varepsilon_{z}=\varepsilon_{r}$ (continuous black line 4). The lower threshold $\beta_{C}=0.5$ (top), $\beta_{C} \approx 0.74$ (bottom).

\section{CONCLUSION}

The main conclusions of this study are the following. In general, the usage of an anisotropic material can result in much larger generated field in comparison to the isotropic case with the same parameters of the bunch and the geometry of the problem.

When the bunch flies from a vacuum into the anisotropic dielectric, similar to the case of the isotropic dielectric, the DWA scheme can be realized in a way that transition effects are small. It has been shown that the area of the boundary influence decreases significantly with an increase in both transversal component of the permittivity tensor and the velocity of bunch motion. The magnitudes and frequencies of CR are larger than in the case of the isotropic dielectric if longitudinal component of the permittivity tensor is less than the transversal one. These effects can be of interest for further development of the DWA technique.

When the bunch flies from the anisotropic dielectric into a vacuum, the CTR in the vacuum region can be more significant than in the case of the isotropic dielectric. In contrast to the case of the isotropic dielectric, the CTR can be generated by ultrarelativistic bunches even for large values of components of the permittivity tensor. The CTR effect can be of interest for elaboration of a microwave radiation generator of a new type. It should be also noted that the CTR characteristics can be easily calculated with rather simple formulas without any complicated integrations.

\section{ACKNOWLEDGMENTS}

This work was supported by the Russian Science Foundation (Grant No. 18-72-10137).

[1] B. M. Bolotovskii, Theory of Cerenkov radiation (III), Sov. Phys. Usp. 4, 781 (1962).

[2] W. Gai, P. Schoessow, B. Cole, R. Konecny, J. Norem, J. Rosenzweig, and J. Simpson, Experimental Demonstration of Wake-Field Effects in Dielectric Structures, Phys. Rev. Lett. 61, 2756 (1988).

[3] A. M. Altmark, A. D. Kanareykin, and I. L. Sheinman, Tunable wakefield dielectric-filled accelerating structure, Tech. Phys. 50, 87 (2005).

[4] W. Gai, Advanced accelerating structures and their interaction with electron beams, AIP Conf. Proc. 1086, 3 (2009).

[5] A. Kanareykin, Cherenkov radiation and dielectric based accelerating structures: Wakefield generation, power extraction and energy transfer efficiency, J. Phys. Conf. Ser. 236, 012032 (2010).

[6] K. Bane and G. Stupakov, Terahertz radiation from a pipe with small corrugations, Nucl. Instrum. Methods Phys. Res., Sect. A 677, 67 (2012).

[7] K. Bane and G. Stupakov, Measurements of terahertz radiation generated using a metallic, corrugated pipe, Nucl. Instrum. Methods Phys. Res., Sect. A 844, 121 (2017).

[8] S. Antipov, C. Jing, S. Baryshev, A. Kanareykin, D. Wang, W. Gai, A. Zholents, and M. Fedurin, THz radiation generation in a multimode wakefield structure, in Proceedings of IPAC, Richmond, VA, USA (JACoW, Geneva, Switzerland, 2015), p. 1929, http://accelconf.web.cern .ch/AccelConf/IPAC2015/papers/tupma042.pdf.

[9] S. Antipov, S. V. Baryshev, R. Kostin, S. Baturin, J. Qiu, C. Jing, C. Swinson, M. Fedurin, and D. Wang, Efficient extraction of high power $\mathrm{THz}$ radiation generated by an ultra-relativistic electron beam in a dielectric loaded waveguide, Appl. Phys. Lett. 109, 142901 (2016).

[10] B. O'Shea, G. Andonian, S. Barber, K. L. Fitzmorris, S. Hakimi, J. Harrison, P. D. Hoang, M. J. Hogan, B. Naranjo, O. B. Williams et al., Observation of acceleration and deceleration in gigaelectron-volt-per-metre gradient dielectric wakefield accelerators, Nat. Commun. 7, 12763 (2016).

[11] D. Wang, X. Su, L. Yan, Y. Du, Q. Tian, Y. Liang, L. Niu, W. Huang, W. Gai, C. Tang, and S. Antipov, Phase control with two-beam interferometry method in a terahertz dielectric wakefield accelerator, Appl. Phys. Lett. 111, 174102 (2017).

[12] S. N. Galyamin, A. V. Tyukhtin, S. Antipov, and S. S. Baturin, Terahertz radiation from an ultra-relativistic charge exiting the open end of a waveguide with a dielectric layer, Opt. Express 22, 8902 (2014).

[13] S. Antipov, C. Jing, A. Kanareykin, J.E. Butler, V. Yakimenko, M. Fedurin, K. Kusche, and W. Gai, Experimental demonstration of wakefield effects in a $\mathrm{THz}$ 
planar diamond accelerating structure, Appl. Phys. Lett. 100, 132910 (2012).

[14] A. M. Cook, R. Tikhoplav, S. Y. Tochitsky, G. Travish, O. B. Williams, and J. B. Rosenzweig, Observation of Narrow-Band Terahertz Coherent Cherenkov Radiation from a Cylindrical Dielectric-Lined Waveguide, Phys. Rev. Lett. 103, 095003 (2009).

[15] G. Andonian, O. Williams, X. Wei, P. Niknejadi, E. Hemsing, J. B. Rosenzweig, P. Muggli, M. Babzien, M. Fedurin, K. Kusche et al., Resonant excitation of coherent Cerenkov radiation in dielectric lined waveguides, Appl. Phys. Lett. 98, 202901 (2011).

[16] R. H. Kochergov and G. V. Sotnikov, Excitement of wakefields with relativistic electron bunch in an anisotropic dielectric waveguide, Ukr. J. Phys. 43, 921 (1998).

[17] I. Sheinman, S. Baturin, and A. Kanareykin, Analysis of a rectangular dielectric-lined accelerating structure with an anisotropic loading, in Proceedings of the 3rd International Particle Accelerator Conference, New Orleans, LA, 2012 (IEEE, Piscataway, NJ, 2012), p. 2769, http://accelconf.web.cern.ch/Accelconf/IPAC2012/papers/ weppp022.pdf.

[18] N. A. Xizhnyak, Artificial anisotropic dielectrics: I, II and III, Zh. Tekh. Fiz. 27, 2006 (1957) [Sov. Phys. Tech. Phys. 2, 1858 (1957)].

[19] S. Yakovenko, L. Yu. Matzui, L. L. Vovchenko, V. V. Oliynyk, V. L. Launetz, and A. V. Trukhanov, Dielectric properties of composite materials containing aligned carbon nanotubes, Inorg. Mater. (USSR) 52, 1198 (2016).

[20] M. I. Kaganov, A beam in a cylindrical waveguide loaded with an anisotropic dielectric, Zh. Exp. Tekh. Fiz. 23, 514 (1953).

[21] V. P. Zrelov, Vavilov-Cherenkov Radiation in High-Energy Physics (Israel Program for Scientific Translations, Jerusalem, 1970).

[22] Ye. L. Burshtein and G. V. Voskresenskii, Radiation of a single charge in a semi-infinite waveguide filled with a dielectric, Zh. Tekh. Fiz. 33, 34 (1963) [Sov. Phys. Tech. Phys. 8, 22 (1963)].

[23] V. A. Balakirev, I. N. Onishchenko, D. Yu. Sidorenko, and G. V. Sotnikov, Excitation of a wake field by a relativistic electron bunch in a semi-infinite dielectric waveguide, Zh. Exp. Teor. Fiz. 120, 41 (2001) [JETR 93, 33 (2001)].

[24] T. Yu. Alekhina and A. V. Tyukhtin, Radiation of a charge in a waveguide with a boundary between two dielectrics, J. Phys. Conf. Ser. 357, 012010 (2012).

[25] T. Yu. Alekhina and A. V. Tyukhtin, Cherenkov-transition radiation in a waveguide with a dielectric-vacuum boundary, Phys. Rev. ST Accel. Beams 15, 091302 (2012).

[26] T. Yu. Alekhina and A. V. Tyukhtin, Self-acceleration of a charge intersecting a boundary surface in a waveguide, Phys. Rev. ST Accel. Beams 16, 081301 (2013).

[27] A. A. Grigoreva, A. V. Tyukhtin, V. V. Vorobev, and S. Antipov, Radiation of a charge intersecting a boundary between a bilayer area and a homogeneous one in a circular waveguide, IEEE Trans. Microwave Theory Tech. 66, 49 (2018).

[28] A. A. Grigoreva, A. V. Tyukhtin, V. V. Vorobev, S. N. Galyamin, and S. Antipov, Radiation of a charge flying in a partially loaded dielectric section of a waveguide, Phys. Rev. Accel. Beams 21, 031302 (2018).

[29] V.L. Ginzburg and V. N. Tsytovich, Transition Radiation and Transition Scattering (Hilger, London, 1990), p. 445.

[30] S. N. Galyamin and A. V. Tyukhtin, Electromagnetic field of a moving charge in the presence of a left-handed medium, Phys. Rev. B 81, 235134 (2010).

[31] S. N. Galyamin and A. V. Tyukhtin, Electromagnetic field of a charge traveling into an anisotropic medium, Phys. Rev. E 84, 056608 (2011).

[32] T. Yu. Alekhina and A. V. Tyukhtin, Electromagnetic field of a charge intersecting a cold plasma boundary in a waveguide, Phys. Rev. E 83, 066401 (2011). 